\title{
Exploring detection of nuclearites in a large liquid scintillator neutrino detector
}

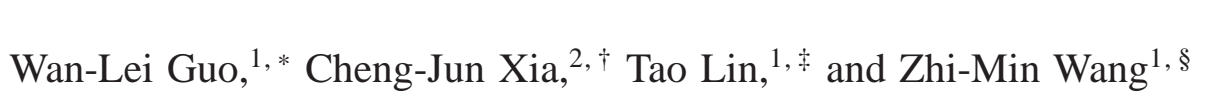 \\ ${ }^{1}$ Institute of High Energy Physics, Chinese Academy of Sciences, \\ P.O. Box 918, Beijing 100049, China \\ ${ }^{2}$ Key Laboratory of Theoretical Physics, Institute of Theoretical Physics, \\ Chinese Academy of Sciences, Beijing 100190, China
}

\begin{abstract}
We take the JUNO experiment as an example to explore nuclearites in the future large liquid scintillator detector. Comparing to the previous calculations, the visible energy of nuclearites across the liquid scintillator will be reestimated for the liquid scintillator based detector. Then the JUNO sensitivities to the nuclearite flux are presented. It is found that the JUNO projected sensitivities can be better than $7.7 \times 10^{-17} \mathrm{~cm}^{-2} \mathrm{~s}^{-1} \mathrm{sr}^{-1}$ for the nuclearite mass $10^{15} \mathrm{GeV} \leq M \leq 10^{24} \mathrm{GeV}$ and initial velocity $10^{-4} \leq \beta_{0} \leq 10^{-1}$ with a 20 year running. Note that the JUNO will give the most stringent limits for downgoing nuclearites with $1.6 \times 10^{13} \mathrm{GeV} \leq M \leq 4.0 \times 10^{15} \mathrm{GeV}$ and a typical galactic velocity $\beta_{0}=10^{-3}$.

PACS numbers: 14.80.-j, 21.65.-f, 95.55.Vj
\end{abstract}

*Electronic address: guowl@ihep.ac.cn
†Electronic address: cjxia@itp.ac.cn
‡Electronic address: lintao@ihep.ac.cn
${ }^{\S}$ Electronic address: wangzhm@ihep.ac.cn 


\section{INTRODUCTION}

Strange quark matter (SQM) is a hypothetical strongly interacting matter composed of roughly equal numbers of $u, d, s$ quarks and a small amount of electrons [1, 2]. It is believed that SQM is the true ground state of quantum chromodynamics, where absolutely stable SQM objects with the baryon number $A$ ranging from that of ordinary nuclei to neutron stars $\left(A \sim 10^{57}\right)$ are expected [3]. The SQM has a little larger density than the saturation density of ordinary nuclear matter, which may be created in various situations, e.g., the hadronization process of the early universe [2], collision of binary compact stars [4, 5], type II supernovae driven by deconfinement phase transition [6], and even heavy ion collisions on Earth [7, 8]. The SQM objects are considered as the cold dark matter candidates and may be presented in the cosmic radiation reaching Earth. Light SQM objects $\left(A<10^{7}\right)$ are usually called strangelets [9-13], while in this work we focus on heavier ones $\left(M>10^{10} \mathrm{GeV}\right)$ known as nuclearites [14, 15]. Based on their special properties, the nuclearite searches have been performed by identifying the seismic activities with an epilinear source on Earth [16] and the moon [17], the ionization tracks in ancient mica [18] and CR39 nuclear track detectors in the MACRO [19, 20], SLIM [21], and Ohya [22] experiments, the bar excitations induced by the thermoacoustic effect in resonant bar detectors [23], the Rutherford backscattering of very heavy nuclei [24-26], the signatures of gravitational lensing caused by massive nuclearites [27, 28], and the photons emitted when a nuclearite moves through water in the ANTARES [29] experiment and atmosphere in the future JEM-EUSO [30] experiment, etc. Despite the nonobservation of nuclearites, these experiments are able to constrain the upper limits on the flux of cosmic nuclearites.

The liquid scintillator (LS) as the detection medium in the past neutrino experiments has achieved great successes [31-34]. Now the next generation large LS detectors JUNO [35, 36] and LENA [37] are constructing in China and proposed in Europe, respectively. When a nuclearite passes through the LS medium, the elastic collisions between the nuclearite and ambient LS molecules will result in an overheating track. Many photons from the black-body radiation of this track can be observed by the photomultiplier tubes (PMTs). Therefore the future large LS detectors have the ability to search for nuclearites. A major advantage of the LS detectors is that the LS wavelength shifters can absorb the short wavelength photons and reemit the longer wavelength photons. This feature ensures that the LS detectors can collect more photons from the black-body radiation of the nuclearite track. Here we shall take the JUNO detector as an example to ex- 
plore nuclearites. The JUNO is a 20 kton multipurpose underground LS detector and primarily determines the neutrino mass hierarchy by detecting reactor antineutrinos. The JUNO detector is deployed in a $700 \mathrm{~m}$ underground laboratory and consists of a central detector, a water Cherenkov detector and a muon tracker. The JUNO central detector holds a $20 \mathrm{kton}$ LS which will be in a spherical container with a radius of $17.7 \mathrm{~m} \mathrm{[35]}$. There is $1.5 \mathrm{~m}$ water buff region between about 18000 20-in PMTs, 36000 3-in PMTs and the LS surface.

In this paper, we shall explore nuclearites in the JUNO LS detector and analyze the JUNO detection capability. Comparing to the previous calculations, the visible energy of nuclearite per unit track length in the JUNO LS region will be reestimated in terms of the LS fluorescence quantum yields, the PMT quantum efficiencies and the JUNO detector design. Then we predict the JUNO sensitivities to the nuclearite flux. In Sec. II, we outline the main features of the nuclearite and give the maximal zenith angle below which nuclearites may pass through the Earth rocks and reach the JUNO detector. In Sec. III, the light yield of nuclearites traversing the JUNO LS will be analyzed in detail. In Sec. IV, we present the JUNO sensitivity to the nuclearite flux based on some conditions. Finally, some discussions and conclusions will be given in Sec. V.

\section{THE NUCLEARITE ENERGY LOSS}

The dominant energy loss mechanism for nuclearites passing through matter is elastic or quasielastic collisions with the ambient atoms. As with meteorites, the nuclearite energy loss rate can be written as [14]

$$
\frac{d E}{d x}=-\sigma \rho \beta^{2}
$$

where $\beta$ is its velocity and $\rho$ is the density of the traversed medium. The effective nuclearite cross section $\sigma$ is given by

$$
\sigma= \begin{cases}\pi R_{0}^{2}=\pi\left(3 M / 4 \pi \rho_{N}\right)^{2 / 3} ; & M \geq 8.4 \times 10^{14} \mathrm{GeV}, \\ \pi \AA^{2}=\pi \times 10^{-16} \mathrm{~cm}^{2} ; & M<8.4 \times 10^{14} \mathrm{GeV},\end{cases}
$$

where the nuclearite density is estimated to be $\rho_{N}=3.6 \times 10^{14} \mathrm{~g}^{-1} \mathrm{~cm}^{-3}$ [9] and the nuclearite radius $R_{0}$ can be easily induced from its mass $M$ and density $\rho_{N}$. When the nuclearite radius $R_{0}<1 \AA$ $\left(M<8.4 \times 10^{14} \mathrm{GeV}\right), \sigma$ is dominated by the nuclearite electron atmosphere which is never smaller than the typical atomic size with the radius of $\sim 1 \AA$ [14, 38]. It is worthwhile to stress that the 
right-hand side of Eq. (1) should be replaced by the constant retarding force $-\varepsilon \sigma$ for the subsonic velocity $\beta<\beta_{c}=\sqrt{\varepsilon / \rho}$ with a structural energy density $\varepsilon \approx 10^{9} \mathrm{erg} \mathrm{cm}^{-3}$ [14].

Based on Eqs. (1) and (2), the travel length of a nuclearite depends on its mass $M$, velocity $\beta$ and the medium density $\rho$. Some nuclearites may pass through the Earth rocks and arrive at the JUNO detector which has a $700 \mathrm{~m}$ rock overburden. For the JUNO detectable nuclearites, they will traverse different thicknesses of rocks based on their direction (zenith angle $\theta_{z}$ ). In addition, one should consider the change of the Earth matter density. In terms of the PREM Earth density profile [39], we numerically calculate the maximal zenith angle $\theta_{\max }$ below which nuclearites may reach the JUNO detector, namely its local velocity $\beta_{1}>0$ at the detector level. The corresponding results have been plotted in Fig. 1 for $10^{12} \mathrm{GeV} \leq M \leq 10^{24} \mathrm{GeV}$ and five typical initial velocities $\beta_{0}$ at the ground level. It is clear that nuclearites from the $\theta_{z}=0^{\circ}$ direction can reach the JUNO detector for $M>10^{13} \mathrm{GeV}$ and $10^{-5} \leq \beta_{0} \leq 10^{-1}$. For a typical galactic velocity $\beta_{0}=10^{-3}$, all directional nuclearites can arrive at the detector when $M>2.5 \times 10^{22} \mathrm{GeV}$. Since the Earth density sharply changes between the core and mantle, we can see the knee points at $\theta_{z}=146.9^{\circ}$ in Fig. 1.

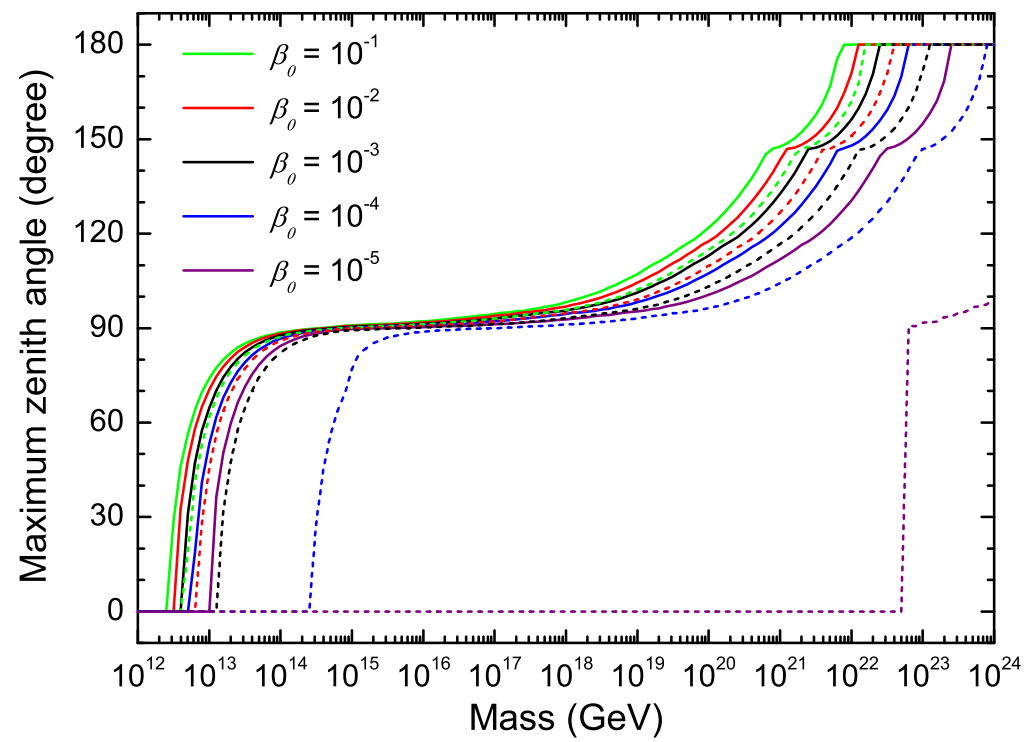

FIG. 1: The maximal zenith angles $\theta_{\max }$ below which the local velocity of nuclearite satisfies $\beta_{1}>0$ (solid lines) and $\beta_{1}>\beta_{\min }$ (dashed lines) for 5 typical initial velocities $\beta_{0}$ at the ground level. In Sec. IV we shall describe the minimal local velocity $\beta_{\min }$. 


\section{THE VISIBLE ENERGY OF NUCLEARITE IN JUNO LS}

When a nuclearite traverses the JUNO LS medium, the LS molecules $\left(\mathrm{C}_{18} \mathrm{H}_{30}\right)$ along the nuclearite path will disintegrate into their constituents because of the nuclearite elastic or quasielastic collisions. These heated atoms will further collide with the ambient LS molecules and generate a hot plasma shockwave [15]. The evolutions of effective temperature $T(t)$ and radius $R(t)$ of the expanding thermal shockwave can be written as [14]

$$
\begin{aligned}
R^{2}(t) & =\sqrt{8} \beta_{1} t R_{0}, \\
T(t) & =m \beta_{1} R_{0} /(\sqrt{8} n t),
\end{aligned}
$$

where $m$ is the LS molecule mass and $n=48$ is the number of submolecular species. Here $\beta_{1}$ denotes the local velocity of nuclearite at the detector level. The expanding cylindrical thermal shockwave can emit lights through the black-body radiation. The corresponding power spectrum is given by

$$
\frac{d p}{d \omega d a}=\frac{\hbar \omega^{3}}{4 \pi^{2} c^{2}} \frac{1}{e^{\hbar \omega / k T}-1}
$$

where $\omega$ is the angular frequency and $a$ denotes the area of shockwave. Then we can deduce the emitted photon numbers per unit track length $d N_{\gamma} / d x$ from the expanding cylindrical shock:

$$
\begin{aligned}
\frac{d N_{\gamma}}{d x} & =\int d \omega \int d t 2 \pi R(t) \frac{d p}{d \omega d a} \frac{1}{\omega} \\
& =\frac{8^{\frac{1}{4}}}{2 \pi} \sqrt{\beta_{1} R_{0}} \int d \omega \int d t t^{\frac{1}{2}} \omega^{2} \frac{1}{e^{\omega / T}-1},
\end{aligned}
$$

where we have used the natural system of units with $\hbar=c=k=1$. Similarly, the total emitted energy $d E_{\gamma} / d x$ can be directly obtained through the replacement $\omega^{2} \rightarrow \omega^{3}$ in Eq. (6).

These emitted photons from the black-body radiation cannot be entirely detected by the JUNO PMTs since they will suffer the absorption, reemission and Rayleigh scattering processes in the JUNO LS [36]. On the other hand, the PMT quantum efficiency is related to the photon wavelength. Therefore one cannot simply use the total emitted energy $d E_{\gamma} / d x$ or the total photon numbers to describe the visible energy in the LS detector. It is convenient for us to calculate the visible energy if the photon electron (pe) efficiency per photon $\epsilon(\lambda)$ is available for the JUNO detector. Based on the LAB, PPO and bis-MSB fluorescence quantum yields [40], we adopt a combined

PMT quantum efficiency curve shape from the Hamamatsu PMT data (400-800 nm) [41] and a fixed $27 \%$ efficiency $(250-400 \mathrm{~nm})$ to calculate $\epsilon(\lambda)$ for the wavelength range $250 \mathrm{~nm} \leq \lambda \leq 800$ 
$\mathrm{nm}$ as shown in Fig. 2, We have assumed an averaged $60 \%$ survival probability of reemitted photons from the detector center to the PMT surface and the 75\% PMT photocathode coverage. It is found that the modeled $\epsilon(\lambda)$ approaches to zero for $\lambda>640 \mathrm{~nm}$. In the absence of the related experimental data, we do not include the contribution of the $\lambda<250 \mathrm{~nm}$ case. Note that it will not significantly affect our final results.

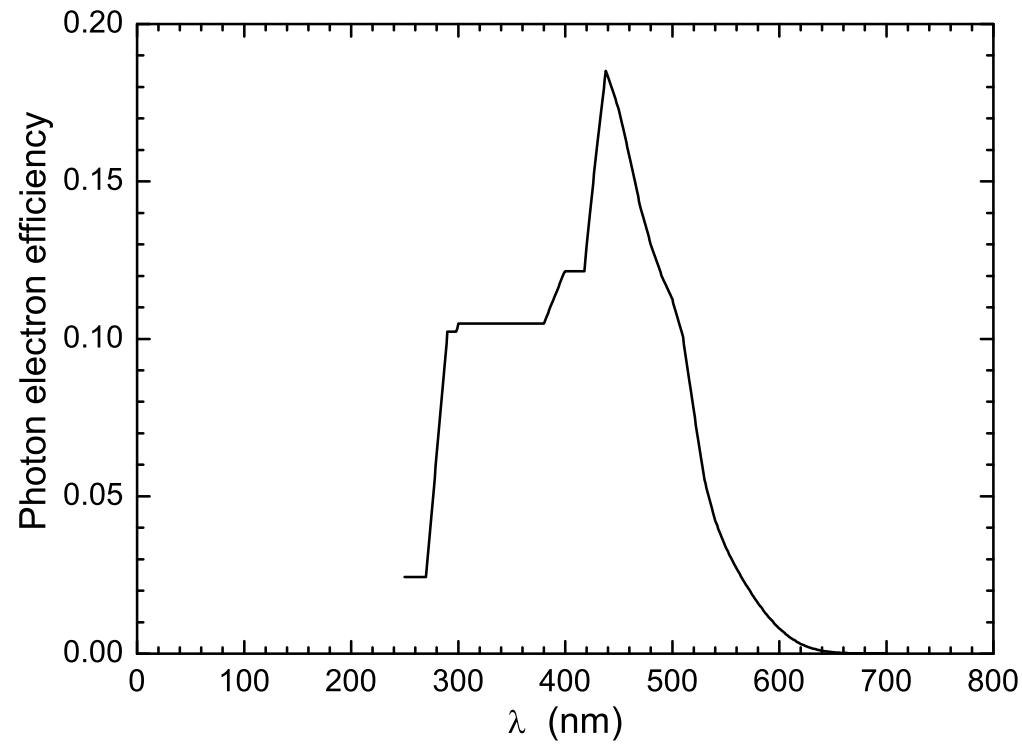

FIG. 2: The modeled photon electron efficiency per photon as a function of the wavelength $\lambda$.

With the help of Eq. (6) and the photon electron efficiency $\epsilon$ in Fig. 2, we can deduce the visible energy of nuclearite per unit track length in the JUNO LS:

$$
\frac{d E_{v i s}}{d x}=\frac{\mathrm{MeV}}{1200 \mathrm{pe}} \frac{8^{\frac{1}{4}}}{2 \pi} \sqrt{\beta_{1} R_{0}} \int d \omega \omega^{2} \epsilon(\omega) \int_{t_{\min }}^{\infty} d t t^{\frac{1}{2}} \frac{1}{e^{\omega / T}-1},
$$

where $t_{\min }$ takes the larger one of $t_{0}=R_{0} /\left(\sqrt{8} \beta_{1}\right)$ and $t_{1}=\left(l / R_{0}\right)^{2} t_{0}$ [14] with the mean free path $l \approx 2.7 \AA$. In Eq. (7), we have simply used that the $1 \mathrm{MeV}$ gamma in the detector center may averagely produce 1200 photon electrons [35]. Based on Eq. (7), one may numerically calculate $d E_{v i s} / d x$ as shown in the left panel of Fig. 3. It is clear that $d E_{v i s} / d x$ does not vary for $M<8.4 \times 10^{14} \mathrm{GeV}$. This is because we have adopted a constant value for $R_{0}$ in Eqs. (3) and (4), i.e., the radius of nuclearite electron atmosphere $1 \AA$. In the right panel of Fig. 3 , we plot the ratio of $d E_{v i s} / d x$ and $d E / d x$ as a function of the local nuclearite velocity $\beta_{1}$. It is found that this ratio is independent of the nuclearite mass $M$ when $M<8.4 \times 10^{14} \mathrm{GeV}$ and $M>1.7 \times 10^{16} \mathrm{GeV}$. For the $M>1.7 \times 10^{16} \mathrm{GeV}$ case, namely $t_{\min }=t_{0}>t_{1}$, one may easily find $d E_{v i s} / d x \propto R_{0}^{2}$ through the variable substitution $t=t^{\prime} R_{0}$. 

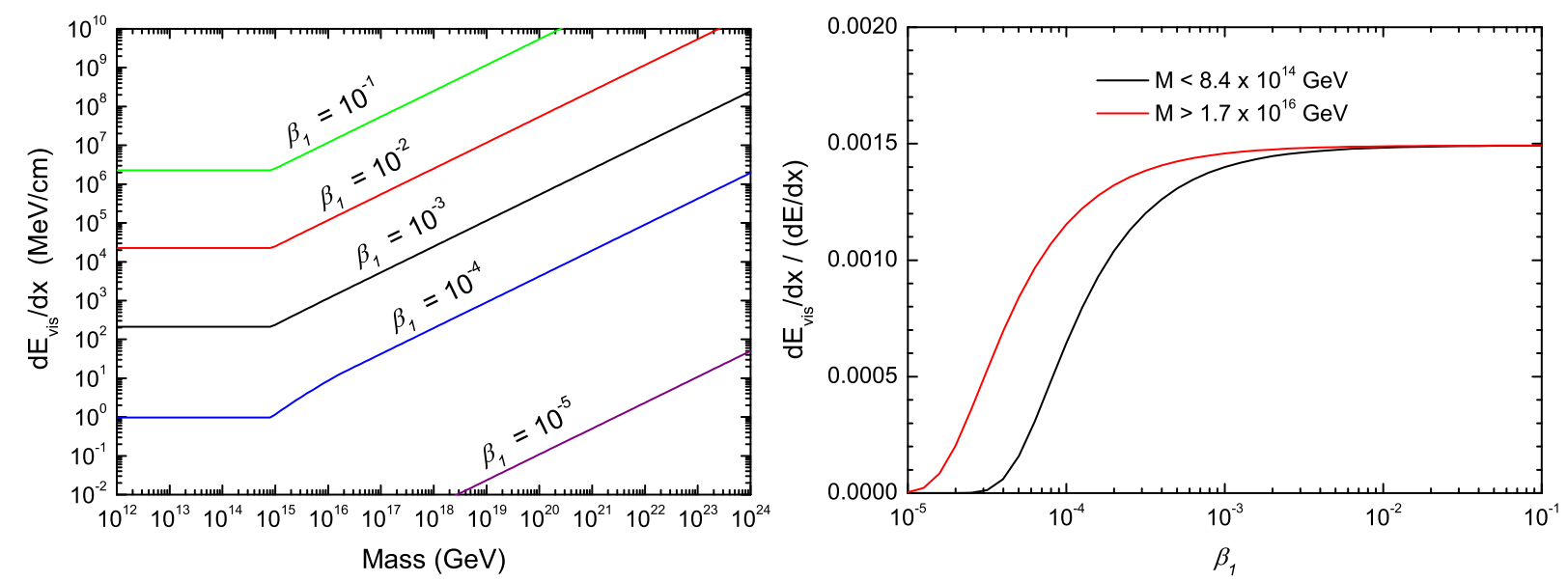

FIG. 3: Left panel: the visible energy of nuclearite per unit track length $d E_{v i s} / d x$ as a function of nuclearite mass for 5 typical $\beta_{1}$; Right panel: the ratio of $d E_{v i s} / d x$ and $d E / d x$ as a function of $\beta_{1}$.

\section{THE EXPECTED JUNO SENSITIVITIES}

The light signals from nuclearites can be recorded when they satisfy the JUNO trigger conditions. Here we assume that the JUNO trigger threshold is $0.5 \mathrm{MeV}$ within a $300 \mathrm{~ns}$ window for the following analyses. Then one may obtain

$$
\beta_{1} \times 300 \mathrm{~ns} \times \frac{d E_{v i s}}{d x} \geq 0.5 \mathrm{MeV} .
$$

With the help of Eqs. (7) and (8), we calculate the minimal local velocity $\beta_{\min }$ as shown in Fig. 4 It is clear that the local velocity $\beta_{1}$ must be larger than $8.7 \times 10^{-6}$ for $10^{12} \mathrm{GeV} \leq M \leq 10^{24} \mathrm{GeV}$. For a fixed initial velocity $\beta_{0}$ at the ground level, the maximal zenith angle $\theta_{\max }$ can be deduced from Eq. (1) and the requirement $\beta_{1}>\beta_{\min }$. In Fig. 11, we have plotted the corresponding $\theta_{\max }$ with dashed lines. It is found that the JUNO may detect all downgoing nuclearites (zenith angle $\theta_{z}<90^{\circ}$ ) with $5.0 \times 10^{15} \mathrm{GeV} \leq M \leq 10^{24} \mathrm{GeV}$ and $10^{-3} \leq \beta_{0} \leq 10^{-1}$. For the $\beta_{0}=10^{-5}$ case, the downgoing nuclearites with $M>6.3 \times 10^{22} \mathrm{GeV}$ can satisfy the $\beta_{1}>\beta_{\text {min }}$ condition.

The expected nuclearite numbers in JUNO can be written as

$$
N_{S}=2 \pi\left(1-\cos \theta_{\max }\right) \phi T_{\text {run }} \pi R_{\text {eff }}^{2}
$$

where $T_{\text {run }}$ is the JUNO running time and $\phi$ is the isotropic nuclearite flux in unit of $\mathrm{cm}^{-2} \mathrm{~s}^{-1} \mathrm{sr}^{-1}$. Here we require that the nuclearite track length in LS region should be larger than $5 \mathrm{~m}$ and derive the effective JUNO radius $R_{\text {eff }}=\sqrt{(17.7 \mathrm{~m})^{2}-(5 \mathrm{~m} / 2)^{2}}=17.52 \mathrm{~m}$. Then the $90 \%$ confidence level 


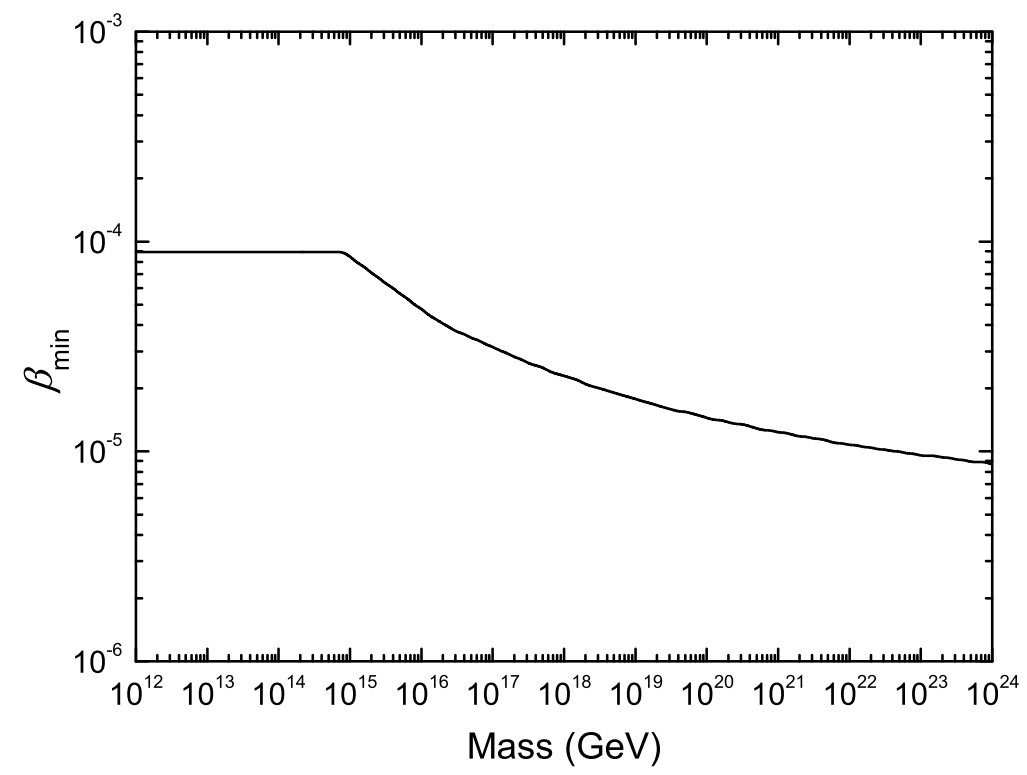

FIG. 4: The minimal local velocity $\beta_{\min }$ above which nuclearites can satisfy the assumed JUNO trigger.

(C.L.) upper limit $N_{90}$ to the expected $N_{S}$ can be derived through the following formula [42, 43]:

$$
90 \%=\frac{\int_{N_{S}=0}^{N_{90}} L\left(N_{\mathrm{obs}} \mid N_{S}\right) d N_{S}}{\int_{N_{S}=0}^{\infty} L\left(N_{\mathrm{obs}} \mid N_{S}\right) d N_{S}}
$$

with the Poisson-based likelihood function

$$
L\left(N_{\mathrm{obs}} \mid N_{S}\right)=\frac{\left(N_{S}+N_{B G}\right)^{N_{\mathrm{obs}}}}{N_{\mathrm{obs}} !} e^{-\left(N_{S}+N_{B G}\right)} .
$$

To estimate the JUNO sensitivities to the nuclearite flux $\phi$, we assume the background number $N_{B G}=0$ and take the observed event number $N_{\text {obs }}=N_{B G}=0$ for a $20 \mathrm{yr}$ running. With the help of Eqs. (9) and (10), we plot the 90\% C.L. flux upper limits (solid lines) for five typical initial velocities $\beta_{0}$ as shown in the left panel of Fig. 5. It is clear that the JUNO sensitivities are better than $7.7 \times 10^{-17} \mathrm{~cm}^{-2} \mathrm{~s}^{-1} \mathrm{sr}^{-1}$ for $10^{15} \mathrm{GeV} \leq M \leq 10^{24} \mathrm{GeV}$ and $10^{-4} \leq \beta_{0} \leq 10^{-1}$. The JUNO is only sensitive to a narrow parameter space of the $\beta_{0}=10^{-5}$ case because of $\beta_{1}<\beta_{\min }$. In addition, the most optimistic limit (black dotted line) has been also plotted for the $\beta_{0}=10^{-3}$ case where we only require $\beta_{1}>0$ and take $R_{\text {eff }}=17.7 \mathrm{~m}$.

To compare with the MACRO [20], ANTARES [29], SLIM [21] and Ohya [22] experimental results, we calculate the JUNO upper limit on the downgoing nuclearites in the $\beta_{0}=10^{-3}$ case. Our numerical results are presented in the right panel of Fig. 5. It is clear that the JUNO sensitivity is far better than the MACRO, SLIM and Ohya limits. Note that the JUNO will give the most stringent limit in the range of $1.6 \times 10^{13} \mathrm{GeV} \leq M \leq 4.0 \times 10^{15} \mathrm{GeV}$. In the most optimistic case 

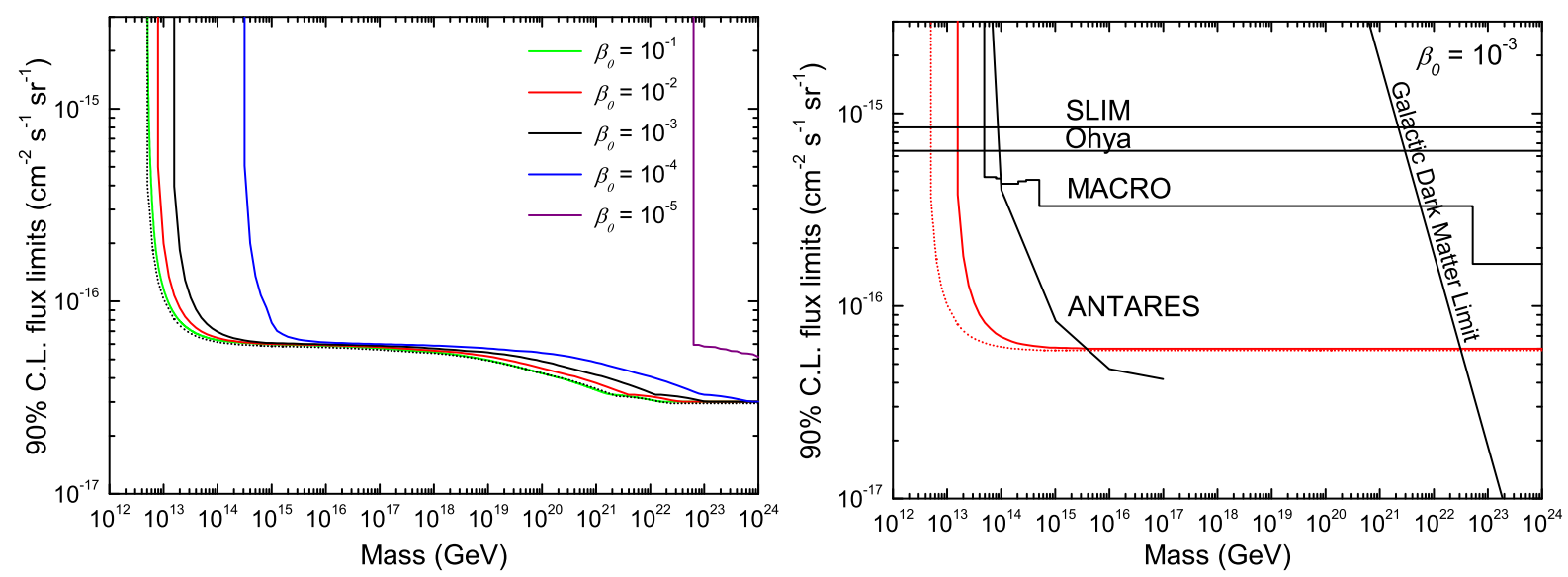

FIG. 5: The JUNO 90\% C.L. flux upper limits to the all direction (left) and downgoing (right) nuclearites with the local velocity $\beta_{1}>\beta_{\min }$ and the effective LS radius $R_{\mathrm{eff}}=17.52 \mathrm{~m}$ for a $20 \mathrm{yr}$ running. The black and red dotted lines describe the JUNO most optimistic limits with $\beta_{1}>0$ and $R_{\text {eff }}=17.7 \mathrm{~m}$ in the initial velocity $\beta_{0}=10^{-3}$ case.

(red dotted line), the above range can be extended to $5.0 \times 10^{12} \mathrm{GeV} \leq M \leq 4.0 \times 10^{15} \mathrm{GeV}$. Here we have also plotted the galactic dark matter (DM) upper limit $\phi_{\max }=\rho_{\mathrm{DM}} \beta_{0} /(2 \pi M)$ [14, 20] where nuclearites are assumed to contribute all of the local DM density $\rho_{\mathrm{DM}}=0.39 \mathrm{GeV} \mathrm{cm}^{-3}$ [44]. For $M>3.1 \times 10^{22} \mathrm{GeV}$, the galactic DM limit is dominant. In the future, the JEM-EUSO experiment will give a more stringent limit $\phi<10^{-20} \mathrm{~cm}^{-2} \mathrm{~s}^{-1} \mathrm{sr}^{-1}$ for $M>5 \times 10^{22} \mathrm{GeV}$ [30].

\section{DISCUSSIONS AND CONCLUSIONS}

As mentioned in Sec. 【II, the photon electron efficiency per photon $\epsilon(\lambda)$ is not considered for the $\lambda<250 \mathrm{~nm}$ range because of the absence of the related experimental data. If these data are available in future, we shall derive the larger $d E_{v i s} / d x$ than those in the left panel of Fig. 3. Then the smaller $\beta_{\min }$ will be expected. The predicted sensitivities (solid lines from $\beta_{1}>\beta_{\min }$ ) in Fig. 5 will approach the most optimistic limits (the corresponding dotted lines from $\beta_{1}>0$ ). It is clear that our results do not change significantly for $M>1.0 \times 10^{14} \mathrm{GeV}$ and $\beta_{0}=10^{-3}$. Note that the nuclearite mass cannot be correctly reconstructed from the incomplete $\epsilon(\lambda)$ when a nuclearite is really detected by the JUNO LS detector. In addition, the JUNO can only give the mass lower bound for very large $d E_{v i s} / d x$ because of the PMT saturation.

In conclusion, we have investigated nuclearites in the JUNO LS detector. Comparing to the previous calculations, the visible energy of nuclearite in the LS has been estimated in detail. Then 
we give the JUNO detectable range of the zenith angle for the nuclearite mass $10^{12} \mathrm{GeV} \leq M \leq$ $10^{24} \mathrm{GeV}$ and five typical initial velocities $\beta_{0}$ at the ground level. Finally, we present the JUNO sensitivities to the nuclearite flux for a $20 \mathrm{yr}$ running. It is found that the JUNO sensitivities to all directional nuclearites are better than $7.7 \times 10^{-17} \mathrm{~cm}^{-2} \mathrm{~s}^{-1} \mathrm{sr}^{-1}$ for $10^{15} \mathrm{GeV} \leq M \leq 10^{24} \mathrm{GeV}$ and $10^{-4} \leq \beta_{0} \leq 10^{-1}$. For the downgoing nuclearites, the expected sensitivities are much better than those from the MACRO, SLIM and Ohya experiments in the case of $\beta_{0}=10^{-3}$. Note that the JUNO will give the most stringent limits for $1.6 \times 10^{13} \mathrm{GeV} \leq M \leq 4.0 \times 10^{15} \mathrm{GeV}$.

\section{Acknowledgments}

We are grateful to Jun Cao, Liang Zhan and Shan-Gui Zhou for their very useful discussions and critical remarks. This work is supported in part by the National Nature Science Foundation of China (Grants No. 11525524, No. 11575201 and No. 11621131001), and the Strategic Priority Research Program of the Chinese Academy of Sciences under Grant No. XDA10010100.

[1] A. R. Bodmer, Phys. Rev. D 4, 1601 (1971). doi:10.1103/PhysRevD.4.1601

[2] E. Witten, Phys. Rev. D 30, 272 (1984). doi:10.1103/PhysRevD.30.272

[3] C. J. Xia, G. X. Peng, E. G. Zhao and S. G. Zhou, Phys. Rev. D 93, no. 8, 085025 (2016). doi:10.1103/PhysRevD.93.085025

[4] J. Madsen, J. Phys. G 28, 1737 (2002) doi:10.1088/0954-3899/28/7/327 [hep-ph/0112153].

[5] A. Bauswein, H.-T. Janka, R. Oechslin, G. Pagliara, I. Sagert, J. Schaffner-Bielich, M. M. Hohle and R. Neuhauser, Phys. Rev. Lett. 103, 011101 (2009) doi:10.1103/PhysRevLett.103.011101 [arXiv:0812.4248 [astro-ph]].

[6] H. Vucetich and J. E. Horvath, Phys. Rev. D 57, 5959 (1998) doi:10.1103/PhysRevD.57.5959 [astro-ph/9802363].

[7] K. Borer et al., Phys. Rev. Lett. 72, 1415 (1994). doi:10.1103/PhysRevLett.72.1415

[8] R. M. Weiner, Int. J. Mod. Phys. E 15, 37 (2006) doi:10.1142/S0218301306004004 [hep-ph/0507115].

[9] S. A. Chin and A. K. Kerman, Phys. Rev. Lett. 43, 1292 (1979). doi:10.1103/PhysRevLett.43.1292

[10] E. Farhi and R. L. Jaffe, Phys. Rev. D 30, 2379 (1984). doi:10.1103/PhysRevD.30.2379

[11] M. S. Berger and R. L. Jaffe, Phys. Rev. C 35, 213 (1987). doi:10.1103/PhysRevC.35.213 
[12] E. P. Gilson and R. L. Jaffe, Phys. Rev. Lett. 71, 332 (1993) doi:10.1103/PhysRevLett.71.332 [hep-ph/9302270].

[13] G. X. Peng, X. J. Wen and Y. D. Chen, Phys. Lett. B 633, 314 (2006) doi:10.1016/j.physletb.2005.11.081 [hep-ph/0512112].

[14] A. De Rujula and S. L. Glashow, Nature 312, 734 (1984). doi:10.1038/312734a0

[15] A. De Rujula, Nucl. Phys. A 434, 605C (1985). doi:10.1016/0375-9474(85)90525-1

[16] E. T. Herrin, D. C. Rosenbaum and V. L. Teplitz, Phys. Rev. D 73, 043511 (2006) doi:10.1103/PhysRevD.73.043511 [astro-ph/0505584].

[17] W. B. Banerdt, T. Chui, E. T. Herrin, D. Rosenbaum and V. L. Teplitz, Adv. Space Res. 37, 1889 (2006). doi:10.1016/j.asr.2005.06.034

[18] P. B. Price, Phys. Rev. D 38, 3813 (1988). doi:10.1103/PhysRevD.38.3813

[19] S. P. Ahlen et al. [MACRO Collaboration], Phys. Rev. Lett. 69, 1860 (1992). doi:10.1103/PhysRevLett.69.1860

[20] M. Ambrosio et al. [MACRO Collaboration], Eur. Phys. J. C 13, 453 (2000) doi:10.1007/s100520050708 [hep-ex/9904031].

[21] S. Cecchini et al. [SLIM Collaboration], Eur. Phys. J. C 57, 525 (2008) doi:10.1140/epjc/s10052-0080747-7 [arXiv:0805.1797[hep-ex]].

[22] S. Orito et al., Phys. Rev. Lett. 66, 1951 (1991). doi:10.1103/PhysRevLett.66.1951

[23] P. Astone et al., arXiv:1306.5164 [astro-ph.HE].

[24] J.-G. Lv, S.-J. Liu, N.-Q. Liu, Y.-Q. Li, H.-T. Li, S.-Y. Song, Y. Wu, R.-S. Wu, T.-B. Zhang, P.-Y. Zheng, et al., Prog. Phys. 9, 385 (1988), in Chinese, URL http://www.cnki.com.cn/Article/CJFDTOTAL-WLXJ198904000.htm

[25] S.-J. Liu, B.-Z. Xie, Y. Wu, and X.-W. Tang, High Energ. Phys. Nuc. 13, 103 (1989), in Chinese, URL http://wWw.cnki.com.cn/Article/CJFDTotal-KNWL198902001.htm.

[26] D. M. Lowder, Nucl. Phys. Proc. Suppl. 24B, 177 (1991). doi:10.1016/0920-5632(91)90321-5

[27] A. Barnacka, J. F. Glicenstein and R. Moderski, Phys. Rev. D 86, 043001 (2012) doi:10.1103/PhysRevD.86.043001 [arXiv:1204.2056][astro-ph.CO]].

[28] K. Griest, A. M. Cieplak and M. J. Lehner, Astrophys. J. 786, no. 2, 158 (2014) doi:10.1088/0004637X/786/2/158 [arXiv:1307.5798 [astro-ph.CO]].

[29] G. E. Pavalas [ANTARES Collaboration], PoS ICRC 2015, 1060 (2016).

[30] M. Bertaina et al. [JEM-EUSO Collaboration], Exper. Astron. 40, no. 1, 253 (2015). 
doi:10.1007/s10686-014-9375-4

[31] C. L. Cowan, F. Reines, F. B. Harrison, H. W. Kruse and A. D. McGuire, Science 124, 103 (1956). doi:10.1126/science.124.3212.103

[32] K. Eguchi et al. [KamLAND Collaboration], Phys. Rev. Lett. 90, 021802 (2003) doi:10.1103/PhysRevLett.90.021802 [hep-ex/0212021].

[33] C. Arpesella et al. [Borexino Collaboration], Phys. Rev. Lett. 101, 091302 (2008) doi:10.1103/PhysRevLett.101.091302 [arXiv:0805.3843 [astro-ph]].

[34] F. P. An et al. [DAYA-BAY Collaboration], Phys. Rev. Lett. 108, 171803 (2012) arXiv:1203.1669 [hep-ex]].

[35] F. An et al. [JUNO Collaboration], J. Phys. G 43, no. 3, 030401 (2016) doi:10.1088/09543899/43/3/030401 [arXiv:1507.05613 [physics.ins-det]].

[36] Z. Djurcic et al. [JUNO Collaboration], arXiv:1508.07166 [physics.ins-det].

[37] M. Wurm et al. [LENA Collaboration], Astropart. Phys. 35, 685 (2012) doi:10.1016/j.astropartphys.2012.02.011 [arXiv:1104.5620 [astro-ph.IM]].

[38] D. Bakari et al., hep-ex/0004019.

[39] A. M. Dziewonski and D. L. Anderson, Phys. Earth Planet. Inter. 25, 297 (1981).

[40] C. Buck, B. Gramlich and S. Wagner, JINST 10, no. 09, P09007 (2015) doi:10.1088/17480221/10/09/P09007 [arXiv:1509.02327[physics.ins-det]].

[41] http://wWw.hamamatsu.com/resources/pdf/etd/PMT_TPMZ0002E.pdf

[42] T. Tanaka et al. [Super-Kamiokande Collaboration], Astrophys. J. 742, 78 (2011) arXiv:1108.3384 [astro-ph.HE]].

[43] W. L. Guo, Z. L. Liang and Y. L. Wu, Nucl. Phys. B 878, 295 (2014) [arXiv:1305.0912 [hep-ph]]; W. L. Guo, JCAP 1601, no. 01, 039 (2016) doi:10.1088/1475-7516/2016/01/039 [arXiv:1511.04888 [hep-ph]].

[44] K. A. Olive et al. [Particle Data Group Collaboration], Chin. Phys. C 38, 090001 (2014). doi:10.1088/1674-1137/38/9/090001 